\title{
ARMO - Adaptive Road Map Optimization for Large Robot Teams
}

\author{
A. Kleiner, D. Sun and D. Meyer-Delius
}

\section{Linköping University Post Print}

N.B.: When citing this work, cite the original article.

(C2011 IEEE. Personal use of this material is permitted. However, permission to reprint/republish this material for advertising or promotional purposes or for creating new collective works for resale or redistribution to servers or lists, or to reuse any copyrighted component of this work in other works must be obtained from the IEEE.

A. Kleiner, D. Sun and D. Meyer-Delius, ARMO - Adaptive Road Map Optimization for Large Robot Teams, 2011, IEEE/RSJ Int. Conf. on Intelligent Robots and Systems (IROS), 3276-3282.

http://dx.doi.org/10.1109/IROS.2011.6048339

Postprint available at: Linköping University Electronic Press

http://urn.kb.se/resolve?urn=urn:nbn:se:liu:diva-72519 


\title{
ARMO: Adaptive Road Map Optimization for Large Robot Teams
}

\author{
Alexander Kleiner*, Dali Sun* and Daniel Meyer-Delius*
}

\begin{abstract}
Autonomous robot teams that simultaneously dispatch transportation tasks are playing more and more an important role in present logistic centers and manufacturing plants. In this paper we consider the problem of robot motion planning for large robot teams in the industrial domain. We present adaptive road map optimization (ARMO) that is capable of adapting the road map in real time whenever the environment has changed. Based on linear programming, ARMO computes an optimal road map according to current environmental constraints (including human whereabouts) and the current demand for transportation tasks from loading stations in the plant. For detecting dynamic changes, the environment is describe by a grid map augmented with a hidden Markov model (HMM). We show experimentally that ARMO outperforms decoupled planning in terms of computation time and time needed for task completion.
\end{abstract}

\section{INTRODUCTION}

Recent trends in logistics and manufacturing clearly indicate an increasing demand for flexibility, modularity, and re-configurability of material flow systems. Whereas in the past plant installations have been used for decades without change, nowadays product life cycles and the demand for product variety rely on innovative technologies that allow to flexibly reconfigure automation processes without reducing their availability. Therefore, distributed and self-organized systems, such as teams of robots that autonomously organize transportation tasks, are playing an increasingly important role in present logistic centers and manufacturing plants.

Besides the task assignment problem, i.e., allocating robots to different tasks [1], another challenge in this domain is to efficient coordinate the simultaneous navigation of large robot teams in confined and cluttered environments. In general, multiple robot motion planning can be solved by either considering the joint configuration space of the robots [2] or by deploying decoupled techniques that separate the problems of motion planning and coordination [3]. Whereas the first approach is intractable for large robot teams since the dimension of the joint configuration space grows linearly and thus the search space grows exponentially with increasing number of robots, the second approach yields typically sub-optimal solutions, for example, requiring the robots to perform larger detours in order to avoid collisions. Road map planners are a popular method for single robot planning in static environments [4] that compute during a pre-processing phase a connectivity graph in free configuration space that is then used for efficient path planning during runtime. However, dynamic domains, such as industrial environments,

\footnotetext{
* Department of Computer Science, University of Freiburg, Georges-Koehler-Allee 52, 79110 Freiburg, Germany, \{kleiner,sun,meyerdel\}@informatik.uni-freiburg.de
}

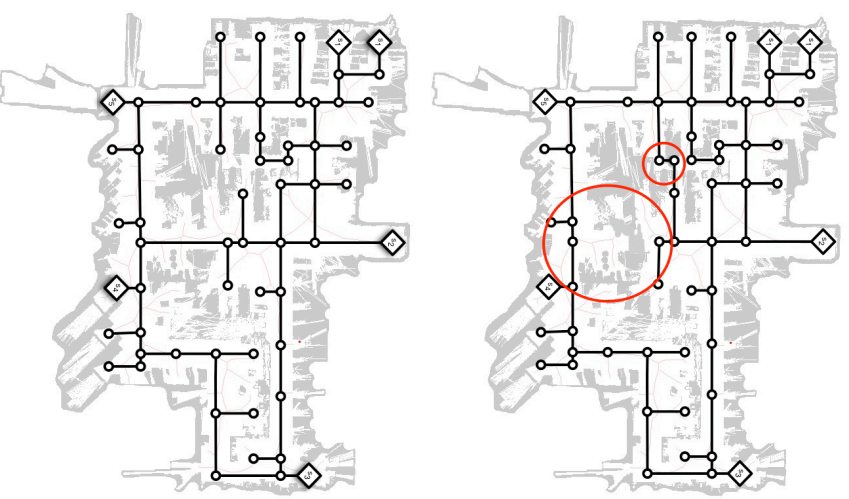

Fig. 1. Motivating example: In industrial environments the map can locally change due to replaced objects, such as pallets, as well as gathering humans. Adaptive road map optimization facilitates the simultaneous navigation planning of large robot teams while respecting these changes.

are more challenging due to permanent changes in the environment, e.g., due to the placement and removal of objects such as pallets and boxes, and the co-location of human workers.

In this paper we present adaptive road map optimization (ARMO) for large robot teams that is capable of adapting the road map in real time whenever the environment has changed. In short, the planner computes an optimal road map according to current environmental constraints (including human whereabouts) and the current demand for transportation tasks from the loading stations. We describe the environment of the robot with a spatial grid map in which a hidden Markov model (HMM) is used to represent dynamic changes. From the continuously updated grid map the computation of a Voronoi Graph [5] is triggered whenever significant changes have been detected. The Voronoi graph, representing free space connectivity, is taken as a starting point to extract road segments (as shown in Figure 1) for the final road map. We use a Linear Programming (LP) approach for computing the optimal configuration of these segments with respect to minimal travel costs and maximal compactness of the network. Figure 1 depicts the re-arrangement of the road map after local changes of the environment have been detected. We show experimentally that ARMO outperforms decoupled planning in terms of computation time and time needed for task completion.

Kallman et al. used dynamic roadmaps for online motion planning based on Rapidly-exploring Random Trees (RRTs) [6]. Velagapudi et al. introduced a distributed version of prioritized planning for large teams where each robot plans simultaneously and re-plans in case a conflict has been detected [7]. Berg et al. presented a method for road 
map based motion planning in dynamic environments [8]. In contrast to our method, which learns changes of the environment online, their approach discriminates between static and dynamic objects, e.g. walls and robots, in advance, which might fail when also portions of the map have to be considered as dynamic. Bellingham et al. proposed a method for solving the cooperative path planning for a fleet of UAVs [9]. They formulate the task allocation problem as a mixed-integer linear program (MILP). Sud et al. developed an approach for path planning of multiple virtual agents in complex dynamic scenes [10]. They utilize first- and secondorder Voronoi diagrams as a basis for computing individual agent paths. While computational efficient, their method does not focus on optimizing the global efficiency of the multi agent team.

The reminder of this paper is organized as follows. In Section II the problem is formally described and in Section III a description of the target system is provided. In Section IV the method for dynamically updating the grid map, and in Section $\mathrm{V}$ the algorithm for adaptively recomputing the road map are described. In Section VI results from experiments are presented and we finally conclude in Section VII.

\section{PROBLEM FORMULATION}

We consider the problem of coordinating the execution of delivery tasks by a team of autonomous robots, e.g., the transportation of crates containing goods, between a set of fixed stations $\mathcal{S}$. For each delivery tasks $d^{k l} \in \mathcal{D}(t)$ a robot has to be assigned to finalize the delivery by transporting the corresponding crate from station $k \in \mathcal{S}$ to station $l \in \mathcal{S}$. We assume that the assignment problem has been solved (e.g. as shown in our previous work [1]), and hence restrict our attention to the problem of solving the multiple robot motion planning problem as defined in the following. Let $\mathcal{R}=\left\{R_{1}, R_{2}, \ldots, R_{n}\right\}$ be the set of $n$ robots navigating simultaneously on a two-dimensional grid map. During planning, each robot has a start configuration $s_{i} \in \mathcal{C}_{\text {free }}$ and a goal configuration $g_{i} \in \mathcal{C}_{\text {free }}$, where $\mathcal{C}_{\text {free }}$ is the subset of configurations robots can take on without colliding with static obstacles. Note that in our case these configurations directly map to locations and orientations on the discrete grid map which are collision free given the footprint of the robot. The problem is to compute for each robot $R_{i} \in \mathcal{R}$ a path $\pi_{i}:\left[0, T_{i}\right] \rightarrow \mathcal{C}_{\text {free }}$ such that $\pi_{i}(0)=s_{i}$ and $\pi_{i}\left(T_{i}\right)=g_{i}$ which is free of collisions with the trajectory $\pi_{j}$ of any other robot $j \neq i$. Note that $T_{i}$ denotes the individual path length of robot $R_{i}$.

We consider environments with dynamic obstacles such as pallets and larger crates that might change their locations over time. Therefore, $\mathcal{C}_{\text {free }}$ is a function of time which we
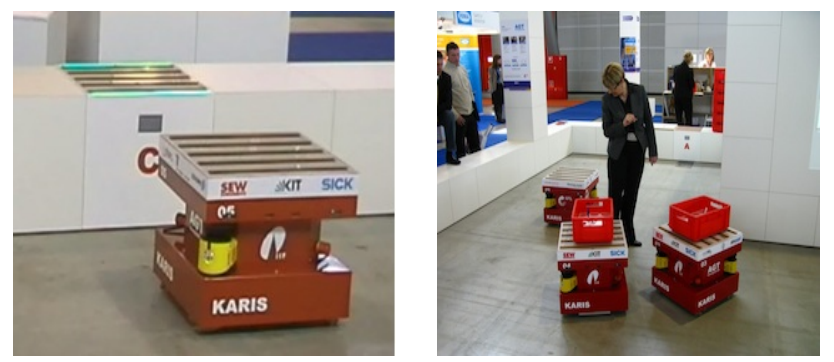

Fig. 2. The target system: Robots equipped with convoyer and RFID reader for autonomously handling transportation tasks: (a) approaching a station for loading. (b) safe navigation among humans.

denote by $\mathcal{C}_{\text {free }}(t)$. Note that we assume that $\mathcal{C}_{\text {free }}$ is static during each planning cycle.

\section{SYSTEM OVERVIEW}

Our system is based on the KARIS (Kleinskalige Autonomes Redundantes Intralogistiksystem) [11] platform developed by a joint effort of several companies and universities of the "Intralogistic Network" in south Germany. The long-term goal of this project is to deploy hundreds of these elements to solve tasks in intra-logistics and production, such as autonomously organizing the material flow between stations. The element has a size of $50 \times 50 \mathrm{~cm}$, a payload of $60 \mathrm{~kg}$, and is capable to recharge its batteries via contactless rechargers let into the ground. Furthermore, it contains a high precision mechanism for enabling automatic docking maneuvers, either with other elements or a loading station. Each element is equipped with a holonomic drive to facilitate docking behaviors and a conveyor for loading and unloading crates when docked with a loading station. The convoyer has an integrated RFID reader for directly reading from the crates their destination, e.g. the target station ID, when they are placed on the conveyer.

For the purpose of autonomous navigation the element is equipped with two SICK S300 laser range finders (LRFs) mounted in two opposing corners, wheel odometry, and an inertial measurement unit (IMU). Navigation is based on grid maps, which are generated from data collected by once steering a single robot manually through the environment. We use Monte-Carlo localization [12] with wheel odometry, IMU, and range readings from the two LRFs for localizing robots on the grid map. Furthermore, the typical hybrid architecture is deployed consisting of two components, which are a deliberative planning layer based on the grid map and a reactive safety layer based on LRF data directly. Figure 2 depicts the demonstration of the system during the Logimat fair in Stuttgart 2010. At the current stage, the system is capable of safe autonomous navigation in human workspaces for team sizes of up to four robots. 


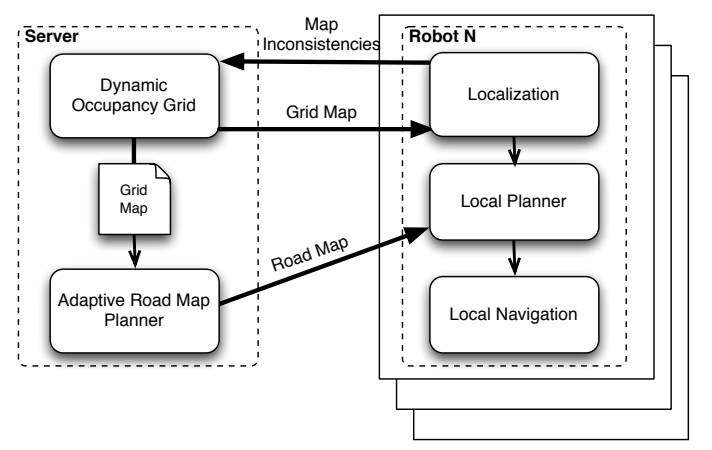

Fig. 3. System Overview

The work presented in this paper has the goal to extend the planning system for the simultaneous navigation of large robot teams in dynamically changing environments. Figure 3 depicts the overall system architecture and modules of the considered extension. The localization module reports inconsistencies between sensor observations and the current grid map to the Dynamic Occupancy Grid Module (see Section IV) which computes an updated version of the grid map. The updated grid map is published to the localization module of each robot, and also to the Adaptive Road Map Planner (see Section V) that computes a new road map, which is then published to the local planner of each robot. The local planner computes then based on the road map a path that is executed by the navigation module. The overview does not contain the mechanism for task allocation, i.e., to assign robots to delivery taks. In the current system this task is solved by the contract net protocol [13], however, also more sophisticated approaches, such as the one presented in our previous work [1] can be deployed.

\section{DYNAMIC OCCUPANCY GRID MAPS}

In this section we describe the procedure for dynamically updating the occupancy grid map when changes in the environment occur. We assume that a map of the entire environment has been generated by an appropriate method for simultaneous localization and mapping (SLAM) in advance [14]. Our method temporarily updates this representation when inconsistencies between laser observations and the map have been detected. For this purpose we combine a conventional occupancy grid map with a hidden Markov model (HMM) that represents the belief about the occupancy and occupancy change probabilities for each cell in the grid. Standard occupancy grid maps [15] are a special case of our representation in which the probabilities of change are zero.

Our model requires the specification of an observation model, the initial state distribution, and the state transition model for each cell. The observation model $p\left(z_{t} \mid c_{t}\right)$ represents the likelihood of observing a cell $c$ as free or occupied

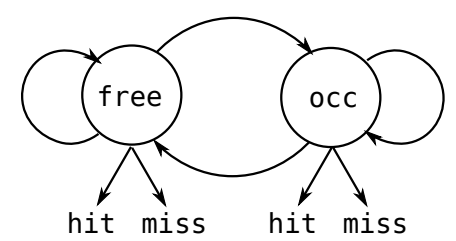

Fig. 4. Graphical model characterizing the state transition probabilities and observation model of a cell in a dynamic occupancy grid.

given the actual state of the cell. In this paper, we consider only observations obtained with a laser range scanner. The cells in the grid that are covered by a superimposed laser beam are determined using a ray-tracing operation. All cells covered by the beam are considered as miss, whereas the cells covered by the endpoint of the beam are considered as hit. We assume that this observation model is equal for each cell and given beforehand.

The initial state distribution $p\left(c_{0}\right)$ specifies the a priori occupancy probability of a cell. The key component in our model, however, are the state transition probabilities $p\left(c_{t} \mid c_{t-1}\right)$ that describe how the occupancy state of cells changes between two consecutive time steps. These probabilities allow us to explicitly characterize how the occupancy of the space changes over time. The underlying assumption behind our approach is that changes in the environment are stationary, that is, the state transition probabilities do not change themselves over time. Based on this stationary dynamic assumption and given that a cell can either be free (free) or occupied (occ), we only need to specify two transition probabilities for each cell, namely the probability of changing from occupied to free and the probability of changing from free to occupied. Figure 4 depicts the structure of the HMM behind our approach. The transition probabilities can be efficiently estimated using the forward-backward procedure (see [16]). The main disadvantage of this instance of the expectation-maximization (EM) algorithm for HMMs is that it is an offline approach and requires to store the complete sequence of observations. An alternative, online version of the EM algorithm [17] is used instead.

In order to update the occupancy of a dynamic occupancy grid map as new observations become available, the following Bayesian approach according to a discrete Bayes filter is used:

$$
p\left(c_{t} \mid z_{1: t}\right)=\eta p\left(z_{t} \mid c_{t}\right) \sum_{c_{t-1}} p\left(c_{t} \mid c_{t-1}\right) p\left(c_{t-1} \mid z_{1: t-1}\right),
$$

where $\eta$ is a normalization constant, $p\left(z_{t} \mid c_{t}\right)$ the observation model and $p\left(c_{t} \mid c_{t-1}\right)$ the state transition probability. Using (1) the belief $p\left(c_{t} \mid z_{1: t}\right)$ over the current occupancy state $c_{t}$ of a cell given all the available evidence $z_{1: t}$ up to 
time $t$ can be recursively estimated from the belief at the previous time step. Note that the map update for standard occupancy grids is also a special case of our approach.

\section{ADAPTIVE ROADMAP PLANNER}

In this section we describe the procedure for computing the adaptive road map given a dynamic occupancy grid map, a set of stations $s \in \mathcal{S}$, where $\operatorname{loc}(s)$ denotes the location $\left(x_{s}, y_{s}\right)$ of station $s$ on the grid map, and a set of delivery tasks $\mathcal{D}$, where each $d^{k l} \in \mathcal{D}$ requires the routing of packages from station $k \in \mathcal{S}$ to station $l \in \mathcal{S}$.

\section{A. Computation of the connectivity network}

Our goal is to compute a road map that is optimal in terms of efficiency and compactness for the simultaneous routing of robots executing delivery tasks. For this purpose we first compute the Voronoi graph [5] from the dynamic grid map, which then serves as a basis for computing the connectivity network $\mathcal{C}=(V, E)$ consisting of nodes $v \in V$ that correspond either to station locations $\operatorname{loc}(s)$ or crossings, and edges $e \in E$ that connect all stations and crossings on the map. The computation of $\mathcal{C}$ is carried out by three steps. First, we determine for each tuple $(i, j) \in \mathcal{S} \wedge i \neq j$ the set of alternative paths $A_{i j}$ connecting station $\mathrm{i}$ and $\mathrm{j}$ on the Voronoi graph. Second, according to the method described in [18], we replace each $A_{i j}$ by orthogonal straight lines (either horizontal or vertical) under the constraint that they have to be within a minimum safety distance to obstacles including the maximal extent of robots from their rotational center. Third, we add all straight lines to $E$ while merging parallel lines if they exceed the double size of the robots. Besides station locations $\operatorname{loc}(s)$, for each crossing line a node is created and added to $V$. Finally, we compute for each $e_{i j} \in E$ the maximal number of possible lanes $w_{i j}$ for this connection according to the distance to the nearest obstacle, and the time needed to travel this segment $c_{i j}$ according to its length.

\section{B. Definition of the LP problem}

Based on the connectivity network $\mathcal{C}$, we define our logistics problem similar to the minimum cost flow problem [19], however, with the difference that the number of lanes in both directions between two nodes and thus the capacities are variable. The goal is to find a network structure by which packages are optimally routed between the stations in the network. At each time there exists a set of simultaneous delivery tasks $d^{k l} \in \mathcal{D}(t)$ that require the routing of packages from station $k \in \mathcal{S}$ to station $l \in \mathcal{S}$. We denote by $b^{k l}=b\left(d^{k l}\right)$ the requested throughput rate, i.e., the amount of packages per minute that have to be delivered from station $k$ to station $l$.

Given the connectivity network $C$, we associate with each edge a cost $c_{i j}$, the maximal number of lanes $w_{i j}$ allowed in the real world, and the capacity of a single lane connection $u_{i j}$. Whereas the cost $c_{i j}$ expresses the time needed to travel from $i$ to $j$, capacity $u_{i j}$ expresses the maximal number of robots that can travel on this connection via a single lane at the same time without causing congestions. The number of lanes in both directions between two nodes $i$ and $j$ is expressed by the decision variables $y_{i j}$ and $y_{j i}$, respectively. For example, $y_{i j}=2, y_{j i}=1$ denotes a single lane connection from node $j$ to node $i$ and a double lane from node $i$ to node $j$. The quantity $w_{i j}$ constraints the set of possible assignments to $y_{i j}$ and $y_{j i}$ according to the space available in the the real world. For example, if $w_{i j}=4$, then possible assignments are $(0,0),(0,1),(1,0),(2,1),(1,2)$, $\ldots,(2,2)$. In general, it has to be assured that $y_{i j}+y_{j i} \leq w_{i j}$. Note that there exists the same limit in both directions and thus $w_{i j}=w_{j i}$.

The decision variables $x_{i j}^{k l}$ define the flow assigned to an edge due to the delivery from $k$ to $l$. The total flow $x_{i j}$ has to be bigger or equal to zero and below the maximal flow $u_{i j} y_{i j}$, where $u_{i j}$ is the capacity of a single lane and $y_{i j}$ the number of activated lanes.

We associate for each delivery task $d^{k l}$ the requested throughput with the receptive station nodes. For each node $i \in V, b(i)=b^{k l}$ if $i=k$, i.e., vertex $i$ is a source, and $b(i)=-b^{k l}$ if $i=l$, i.e., vertex $i$ is a sink. All other nodes for which $b(i)=0$ are functioning as transition nodes. The problem formulation can then be stated as follows:

Minimize $\sum_{(i, j)} \sum_{k} \sum_{l} c_{i j} x_{i j}^{k l}+\sum_{(i, j)} u_{i j} y_{i j}$

subject to:

$$
\begin{aligned}
& \sum_{j:(j, i)} x_{i j}^{k l}-\sum_{j:(i, j)} x_{j i}^{k l}= \begin{cases}-b^{k l}(i) & (i=k) \quad \forall i, k, l \\
b^{k l}(i) & (i=l) \quad \forall i, k, l \\
0 & \text { otherwise. }\end{cases} \\
& y_{i j}+y_{j i} \leq w_{i j} \quad \forall(i, j), \\
& x_{i j}^{k l} \geq 0 \quad \forall(i, j), k, l, \\
& \sum_{k} \sum_{l} x_{i j}^{k l} \leq u_{i j} y_{j i} \quad \forall(i, j), k, l \\
& \sum_{j:(i, j)} x_{j i}^{k l} \leq C_{\max } \quad(i \neq k \wedge i \neq l) \quad \forall i, k, l
\end{aligned}
$$

Equation 2 minimizes over the total travel costs and the physical space occupied by the road network. Equation 3 en- 
forces the flow conservation in the network, and Equation 4, Equation 5, and Equation 6 are constraining the maximal number of lanes, minimal and maximal flow, respectively. Finally, Equation 7 ensures that the total flow through crossings does not exceed the maximal crossing capacity $C_{\max }$ which depends on the spacial size of crossings, i.e., how many robots can be located there at the same time. Note that delivery tasks for which the node operates as source or sink have no influence on the capacity.

The above formulation can efficiently be solved by linear programming solvers, such as CPLEX, when defining the decision variables $x_{i j}, y_{i j}$ by continuous values and rounding up the $y_{i j}$ from which then the road map can directly be constructed. Furthermore, we yield for each delivery task $d^{k l}$ a subset of edges from the road map having positive flow assignments $x_{i j}^{k l}>0$. These quantities are directly utilized by the local planner (see Section III) for extracting individual robot plans by finding the shortest path on the road map by the following successor state expansion: For each node $i$, we perform random sampling over all outgoing edges weighted according to their normalized flow values $x_{i j}^{k l}$. If there exists only one edge with $x_{i j}^{k l}>0$ for node $i$, the edge is expanded directly. Finally, the local navigation module follows this plan while coordinating locally at crossings with other robots when needed.

\section{EXPERIMENTAL RESULTS}

The system has been tested in several different environments. Figure 5 depicts some of these environments that were used for the results presented in this paper. The PLANT map has a size of $51 \mathrm{~m} \times 56 \mathrm{~m}$, the $A S E$ map a size of $94 \mathrm{~m} \times 82 \mathrm{~m}$, and the KNO map a size of $88 m \times 43 m$. On each map we defined locations of loading stations: 8 on PLANT, 16 on ASE, and 8 on KNO.

The robot platform shown in Figure 2 has been presented during the Logimat fair in Stuttgart, 2010, where the task of the robot team was to deliver freshly prepared coffee cups to visitors waiting at the delivery stations, and to return used cups back to the coffee kitchen. During this demonstration up to four robots were continuously running for three days without any interruption. The robots were driving in average four kilometers per day without causing collisions or deadlocks. Due to the small team size we utilized for this demonstration a decoupled planning technique together with the local navigation module. In the following a comparison with large robot teams between ARMO and the decoupled technique based on prioritized planning from Berg and colleagues [20] will be presented. In prioritized planning, robot trajectories are planned iteratively after a pre-defined priority scheme. When planning for the $i$ 'th robot

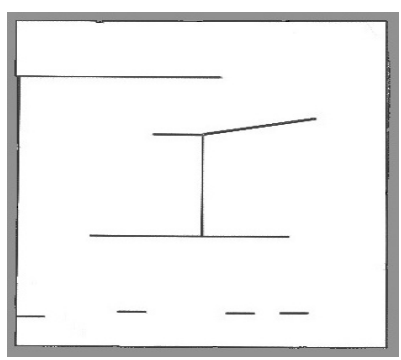

(a)

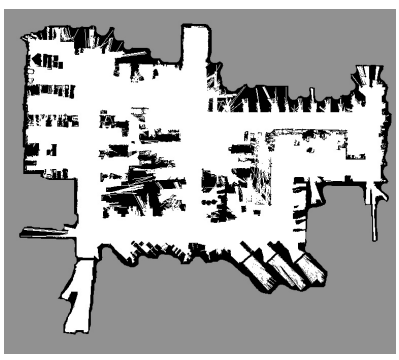

(b)

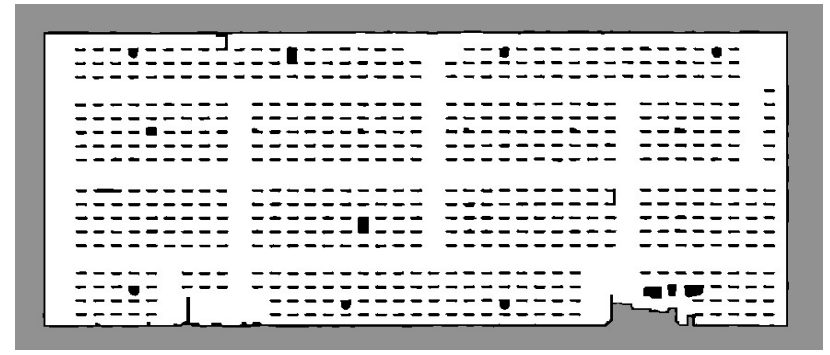

(c)

Fig. 5. Grid maps utilized for experiments: (a) the PLANT map generated from a simulated environment, (b) the $A S E$ map generated in a real logistic center, (c) the $K N O$ map generated in a large distribution center.

trajectories of the i-1 robots that were planned previously are considered as dynamic obstacles. Berg and colleagues define the query distance as the distance for each robot to reach its goal configuration on the shortest path when ignoring the other robots. In order to minimize the maximum of arrival times, priorities are assigned according to this distance: as longer the query distance as higher the priority assigned to a robot. The planner is complete under the assumption that start and goal locations of each robot are so called garage configurations, i.e., configurations that are not part of $\mathcal{C}_{\text {free }}$ of any other robot. The method efficiently avoids the intractable computation of $n$ ! possible priority schemes, however, requires at least $|\mathcal{R}|$ sequential calls of the motion planner.

We utilized the Stage software library [21] for simulating large robot teams. In our experiments we used the same navigation software that is used on the real robots together with a model of our real platform, including the simulation of laser beams and odometry. One advantage of Stage is that it allows to build simulation worlds directly from grid maps that were generated from real environments. For the following experiment we used the grid maps shown in Figure 5.

We generated 100 delivery tasks for each map that were handled by 20,50, and 100 robots during different runs. Table I provides the results from comparing prioritized planning (PRIO) with adaptive road map optimization (ARMO) 


\begin{tabular}{|c|c|c|c|c|c|}
\hline Map & \#Robots & Method & $\# \mathrm{C}$ & avg. v $(\mathrm{m} / \mathrm{s})$ & CTime (s) \\
\hline \multirow{6}{*}{ ASE } & \multirow{2}{*}{20} & ARMO & 1432 & 0.47 & 969 \\
\hline & & PRIO & 2142 & 0.43 & 926 \\
\hline & \multirow{2}{*}{50} & ARMO & 5040 & 0.37 & 545 \\
\hline & & PRIO & 10625 & 0.28 & 631 \\
\hline & \multirow{2}{*}{100} & ARMO & 8307 & 0.3 & 369 \\
\hline & & PRIO & 16983 & 0.2 & 496 \\
\hline \multirow{6}{*}{ PLANT } & \multirow{2}{*}{20} & ARMO & 1471 & 0.42 & 628 \\
\hline & & PRIO & 1346 & 0.38 & 610 \\
\hline & \multirow{2}{*}{50} & ARMO & 5563 & 0.34 & 426 \\
\hline & & PRIO & 11601 & 0.25 & 481 \\
\hline & \multirow{2}{*}{100} & ARMO & 15145 & 0.22 & 383 \\
\hline & & PRIO & 107700 & 0.21 & 874 \\
\hline \multirow{6}{*}{ KNO } & \multirow{2}{*}{20} & ARMO & 506 & 0.38 & 1383 \\
\hline & & PRIO & 5638 & 0.35 & 1346 \\
\hline & \multirow{2}{*}{50} & ARMO & 2951 & 0.31 & 815 \\
\hline & & PRIO & 70799 & 0.16 & 1371 \\
\hline & \multirow{2}{*}{100} & ARMO & 7729 & 0.29 & 513 \\
\hline & & PRIO & 102836 & 0.11 & 1167 \\
\hline
\end{tabular}

TABLE I

COMPARING PRIORITIZED PLANNING (PRIO) WITH ADAPTIVE ROAD MAP OPTIMIZATION (ARMO).

on different maps with different numbers of robots. We measured the number of conflicts (C) of the optimal path in $\mathcal{C}_{\text {free }}$ with trajectories of the other robots. In the case of ARMO these were the situations in which a robot had to wait for other robots before entering a segment, and in the case of prioritized planning these were the situations were robots had to plan around a conflicting path of a higher prioritized robot. Furthermore, we measured the average velocity of all robots (avg. v) and the total time needed by all robots to complete the task (CTime). As can be seen from Table I and Figure 6, while leading to slightly longer completion times for small robot teams, ARMO significantly reduces this time when the team size increases. This is also reflected by the number of conflicts and the average velocities of the robots. Prioritized planning minimizes the final completion time after a heuristically determined order, whereas LP-based planning in ARMO minimizes the global flow of robots, leading to a more efficient distribution of the vehicles over time.

The computation times of both methods were measured in seconds on an Intel DualCore running at $2.13 \mathrm{GHz}$. We measured for prioritized planning with 50 robots an average computation time of $0.03 \pm 0.03$ on PLANT, $0.05 \pm 0.04$ on ASE, and $0.1 \pm 0.17$ on $\mathrm{KNO}$, and with 100 robots $0.1 \pm 0.08$ on PLANT, $0.13 \pm 0.08$ on ASE, and $1.1 \pm 0.7$ on KNO. ARMO required for the road map computation $t_{1}=0.9+$ $t_{2}=0.6$ on PLANT, $t_{1}=0.82+t_{2}=0.84$ on ASE, and $t_{1}=1.2+t_{2}=10.3$ on $\mathrm{KNO}$, where $t_{1}$ is the time for extracting the fully connected graph, and $t_{2}$ the time for solving the LP problem. Within each planning cycle ARMO needed in average only 0.002 on PLANT, 0.004 on ASE,

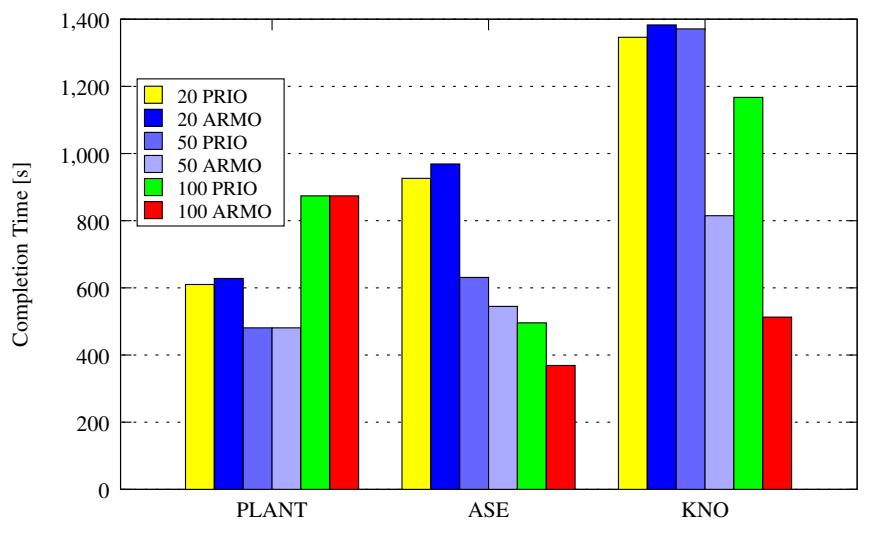

Fig. 6. Comparing the CTime of prioritized planning (PRIO) and adaptive road map optimization (ARMO).

and 0.01 on KNO for any number of robots. In summary, the number of robots has nearly no effect on the computation time needed by ARMO, however, we measured a significant growth of the time needed by prioritized planning. On the contrary, ARMO requires much more time for computing the road map when the environment is very large and complex, such as the KNO map, which however needs only to be performed at low frequency, i.e., when the environment was significantly changed.

We also evaluated ARMO with respect to dynamic changes of the grid map. For this purpose we modified the ASE map step wise by adding successively obstacles that were updated in the map by the dynamic occupancy grid approach. Figure 7 depicts two snapshots taken at successive points in time. As can be seen, the road map adjusts to the changes at the cost of higher completion times. For 100 robots the completion time increased from 378s (no modifications) to $410 \mathrm{~s}$ (first modification) and 420 s (second modification). We performed several more experiments for evaluating the adaptivity of our approach. Also after changing the distribution of delivery tasks between the stations, the road map dynamically adjusted by removing or adding links between the stations. Note that in this case only the LP solver is restarted without re-computing the connectivity network $\mathcal{C}$.

\section{CONCLUSION}

We proposed an adaptive road map planner based on a linear programming formulation which can be used for motion planning of large robot teams in dynamically changing environments. Experimental results have shown that ARMO leads to more efficient multi-robot plans than decoupled techniques while keeping the demand for computational resources low. In fact, the computation time needed by ARMO depends mainly on the complexity of the environment rather 


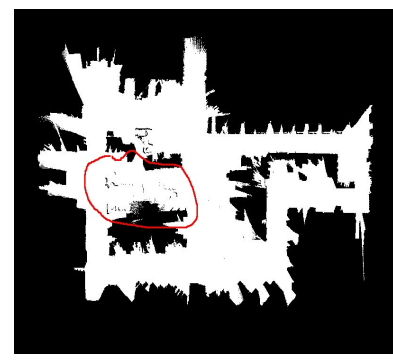

(a)

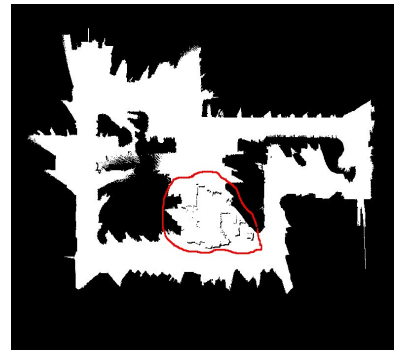

(c)

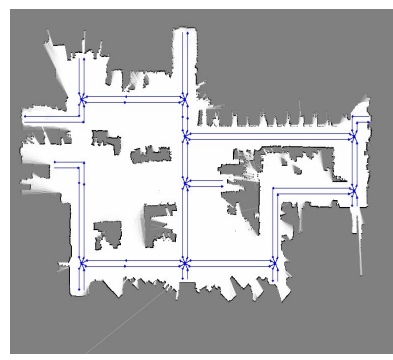

(b)

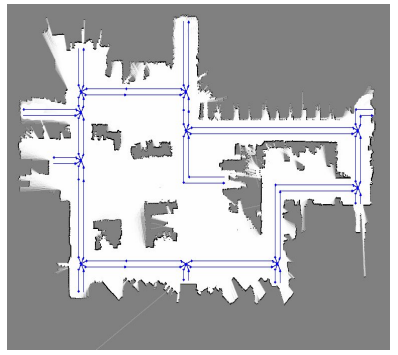

(d)
Fig. 7. Adjustment of the road map according to dynamic changes in the map $(a, c)$ source of disturbance and $(b, d)$ resulting modifications reflected in the road map.

than on the number of robots. We believe that the computation of the road map could further be improved by splitting the map into independent areas that are interconnected via fixed crossing points similar to the stations. Then, only a part of the road map would have to be recomputed after local changes have been detected.

Furthermore, we have shown that ARMO is adaptive to dynamic changes in the map, i.e., the road map is reconstructed accordingly, whereas changes in the map are detected by dynamic grid maps, an extension of conventional grid maps.

We conducted several more experiments and conclude that our method is capable to efficiently solve a wide variety of problems. One restriction of our current implementation is the fact that our road map planner only returns a solution when the overall throughput demanded by the stations can be routed given the environmental constraints, i.e., does not exceed the capacity of the network. One future extension will be to introduce priorities for deliveries and to construct the network from a subset of tasks sampled according to their priority in case the requested throughput is higher than the capacity of the network. Furthermore, when a larger number of real robots is available, ARMO will be used with the real platform deployed in one of the logistic centers of our partners.

\section{REFERENCES}

[1] D. Sun, A. Kleiner, and C. Schindelhauer, "Decentralized hash tables for mobile robot teams solving intra-logistics tasks," in Proc. of the 9th Int. Joint Conf. on Autonomous Agents and Multiagent Systems (AAMAS 2010), Toronto, Canada, 2010, pp. 923-930.

[2] J. Barraquand and J.-C. Latombe, "Robot motion planning: A distributed representation approach," International journal of robotics research, vol. 10, pp. 628-649, 1991.

[3] S. M. LaValle, Planning Algorithms. Cambridge, U.K.: Cambridge University Press, 2006, available at http://planning.cs.uiuc.edu/.

[4] L. Kavraki, P. Svestka, J.-C. Latombe, and M. Overmars, "Probabilistic roadmaps for path planning in high-dimensional configuration spaces," Robotics and Automation, IEEE Transactions on, vol. 12, no. 4, pp. $566-580$, Aug. 1996.

[5] H. Choset, , and B. J., "Sensor-based exploration: The hierarchical generalized voronoi graph," The International Journal of Robotics Research, vol. 19, no. 2, 2000

[6] M. Kallman and M. Mataric, "Motion planning using dynamic roadmaps," in Proc. of the IEEE Int. Conf. on Robotics \& Automation (ICRA), vol. 5, 2004, pp. 4399-4404.

[7] P. Velagapudi, K. Sycara, and P. Scerri, "Decentralized prioritized planning in large multirobot teams," in Intelligent Robots and Systems (IROS), 2010 IEEE/RSJ International Conference on. IEEE, pp. 4603-4609.

[8] J. van den Berg and M. Overmars, "Roadmap-based motion planning in dynamic environments," Robotics, IEEE Transactions on, vol. 21, no. 5, pp. 885-897, 2005.

[9] J. Bellingham, M. Tillerson, M. Alighanbari, and J. How, "Cooperative path planning for multiple uavs in dynamic and uncertain environments," in Decision and Control, 2002, Proceedings of the 41st IEEE Conference on, vol. 3, 2002, pp. 2816 - 2822 vol.3.

[10] A. Sud, E. Andersen, S. Curtis, M. Lin, and D. Manocha, "Realtime path planning in dynamic virtual environments using multiagent navigation graphs," Visualization and Computer Graphics, IEEE Transactions on, vol. 14, no. 3, pp. $526-538,2008$.

[11] H. Hippenmeyer, K. Furmans, T. Stoll, and F. Schönung, "Ein neuartiges Element für zukünftige Materialflusssysteme," Hebezeuge Fördermittel: Fachzeitschrift für Technische Logistik, no. 6, 2009.

[12] F. Dellaert, D. Fox, W. Burgard, and S. Thrun, "Monte carlo localization for mobile robots," in Proc. of the IEEE Int. Conf. on Robotics \& Automation (ICRA), 1998.

[13] R. G. Smith, "The contract net protocol: High-level communication and control in a distributed problem solver," IEEE Transactions on Computers, vol. C-29, no. 12, pp. 1104-1113, 1981.

[14] G. Grisetti, R. Kümmerle, C. Stachniss, U. Frese, and C. Hertzberg, "Hierarchical optimization on manifolds for online $2 \mathrm{~d}$ and $3 \mathrm{~d}$ mapping," in Proc. of the IEEE Int. Conf. on Robotics and Automation (ICRA), Anchorage, AK, USA, May 2010.

[15] H. Moravec and A. Elfes, "High resolution maps from wide angle sonar," in Proc. of the IEEE/RSJ Int. Conf. on Intelligent Robots \& Systems (IROS), 1985.

[16] L. Rabiner, "A tutorial on hidden markov models and selected applications in speech recognition." in Proceedings of the IEEE, vol. 77 (2), 1989, pp. 257-286.

[17] G. Mongillo and S. Deneve, "Online learning with hidden markov models," Neural Computation, vol. 20, pp. 1706-1716, 2008.

[18] X. Décoret and F. X. Sillion, "Street Generation for City Modelling," in Architectural and Urban Ambient Environment, Nantes France, 2002. [Online]. Available: http://hal.inria.fr/inria-00510041/PDF/article.pdf

[19] R. K. Ahuja, T. L. Magnanti, and J. B. Orlin, Network flows: theory, algorithms, and applications. Englewood Cliffs, N.J.: Prentice Hall, 1993, vol. 1.

[20] J. van den Berg and M. Overmars, in Proc. of the IEEE/RSJ Int. Conf. on Intelligent Robots \& Systems (IROS), 2005, pp. 430-435.

[21] R. Vaughan, "Massively multi-robot simulation in stage," Swarm Intelligence, vol. 2, no. 2, pp. 189-208, 2008. 